\title{
Adrenalectomía laparoscópica por patología maligna
}

\author{
A. Vega Vega*, J.M. Canga Presa**, P. Sanz de la Morena***, J.L. de la Cruz Vigo*** \\ *Servicio de Urología de la Obra Hospitalaria Nuestra Señora de Regla, León. **Servicio de Cirugía General del \\ Hospital de León, León. ${ }^{* * *}$ Servicio de Cirugía General de la Obra Hospitalaria Nuestra Señora de Regla, León.
}

Actas Urol Esp 2005; 29 (3): 277-280

\section{RESUMEN}

ADRENALECTOMÍA LAPAROSCÓPICA POR PATOLOGÍA MALIGNA

Existe un consenso generalizado de las bondades de la adrenalectomía laparoscópica respecto a la cirugía abierta en la patología benigna de suprarrenal, por su igual eficacia y menor morbilidad. En lesiones en las que existe sospecha de patología maligna el uso de la laparoscopia es controvertido. Exponemos nuestra experiencia en 6 casos de adrenalectomía laparoscópica en pacientes con sospecha preoperatorio de malignidad, en lesiones confinadas a la glándula. La evolución de estos pacientes en un seguimiento de 24 meses de media (rango 12 a 48 meses) no indicó recidiva tumoral, indicando que cuando la sospecha preoperatoria es de malignidad, sin infiltración de órganos adyacentes el beneficio de la laparoscopia en cuanto a confort postoperatorio se mantiene y no empeora el pronóstico de estos pacientes.

Palabras Clave: Suprarrenal. Laparoscopia. Carcinoma adrenal

\section{ABSTRACT \\ LAPAROSCOPIC ADRENALECTOMY IN ADRENAL CARCINOMA}

There is general agreement on the suitability of the laparoscopic approach for benign adrenal lesions against open procedures because of the efficacy and less morbidity. For suspected adrenal malignancies laparoscopic use is controversial. We report our experience in 6 cases of laparoscopic adrenalectomy in patients with the suspicion of adrenal malignancy confined in the gland. In medium a follow up of 24 months (range 12 to 48 months) we observed no recurrences. We concluded that in a suspected adrenal malignancy organ confined laparoscopic adrenalectomy presents the advantage compared with open surgery of reduced morbidity and similar results in the follow up of the patient.

Keywords: Adrenal. Laparoscopy. Adrenal carcinoma.

$\mathrm{L}^{2}$ a laparoscopia es una alternativa mínimamente invasiva a varias técnicas quirúrgicas urológicas abiertas. Aunque la mayoría de las técnicas urológicas abiertas se han hecho de forma laparoscópica, varias de ellas no se ha probado que presenten ventajas sobre la cirugia abierta convencional. La adrenalectomía laparoscópica, desde la primera descrita en $1992^{1}$ hasta la actualidad, se ha ido estableciendo en muchos centros como el tratamiento estándar en la patología benigna suprarrenal debido a los beneficios que aporta: minima pérdida de sangre, acorta- miento de la estancia hospitalaria y menor tiempo de recuperación con respecto a la adrenalectomía abierta ${ }^{2}$.

Existe un consenso generalizado de la indicación de adrenalectomía laparoscopica en las lesiones benignas de suprarrenal, pero existe controversia en el uso de laparoscopia cuando se sospecha una lesión maligna suprarrenal. Aún no ha conseguido una aceptación generalizada por tratarse de una patología poco frecuente, por lo que las series en la literatura son escasas y con pocos pacientes ${ }^{3}$. En nuestro artículo exponemos 
nuestra experiencia en adrenalectomía en 6 pacientes en los que existía sospecha preoperatoria de tumor maligno o metastático previo a la intervención.

\section{PACIENTES Y MÉTODOS}

Técnica quirúrgica

Aunque existe la posibilidad de un acceso retroperitoneal, en nuestro caso el acceso ha sido siempre transperitoneal, con el paciente en posición de decúbito lateral supino, accediendo al abdomen con una aguja de Veress. Tras la inducción del neumoperitoneo, se insertan 3 o 4 trócares de 5 y $10 \mathrm{~mm}$ en el área subcostal del cuadrante abdominal respectivo, derecho o izquierdo (Fig. 1). El neumoperitoneo se mantiene entre 10 y $12 \mathrm{~mm} \mathrm{Hg}$ durante todo el proceso.

En el lado izquierdo, se abre la flexura esplénica del colon, accediendo al retroperitoneo y se comienza la disección en el polo superior del riñón dejando libre los anclajes posteriores y laterales del bazo en dirección del diafragma. El bazo se retrae medialmente, accediendo a la glándula adrenal. Primero se hace disección del polo superior y posteriormente medial, se visualiza la vena adrenal que se secciona tras obturarla con clips metálicos, y posteriormente se disecciona la parte inferior.

En el lado derecho se diseca y secciona el ligamento triangular del hígado, se retrae el colon transverso y el hígado, se accede al retroperitoneo

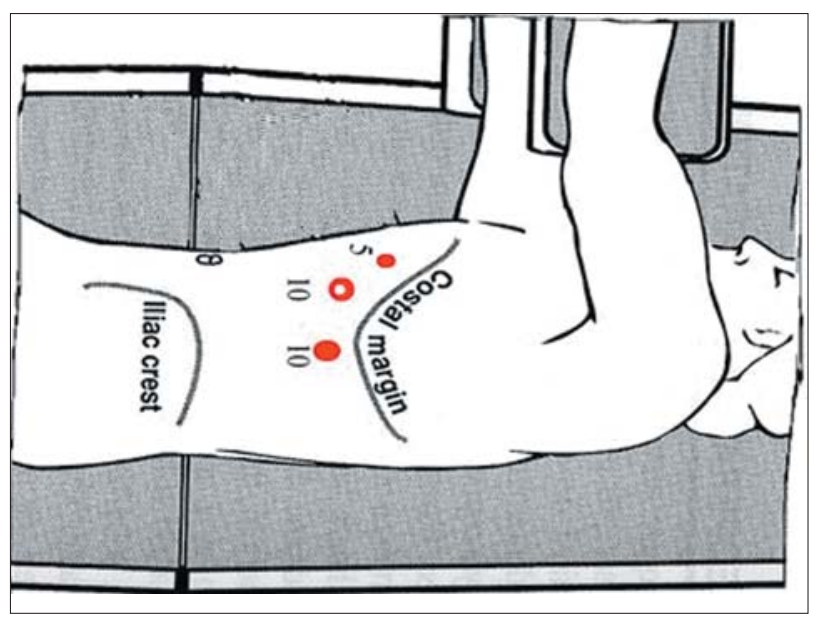

FIGURA 1. Colocación de los trócares para adrenalectomía laparoscópica izquierda, con el paciente en decúbito lateral supino. incidiendo el peritoneo en el borde lateral de la cava inferior. Una vez que se accede a la fascia de Gerota, se identifica el polo superior renal, se diseca la grasa perirrenal superior y próxima a la cava para exponer la glándula adrenal. La disección se comienza por los lados laterales y superiores, se retrae medialmente, se accede a la vena adrenal se colocan clips metálicos y se secciona, posteriormente se libera a nivel inferior (Figs. 2 y 3). Una vez liberada la glándula se introduce en una bolsa para su extracción por uno de los accesos de los trócares de $10 \mathrm{~mm}$, ligeramente ampliado.

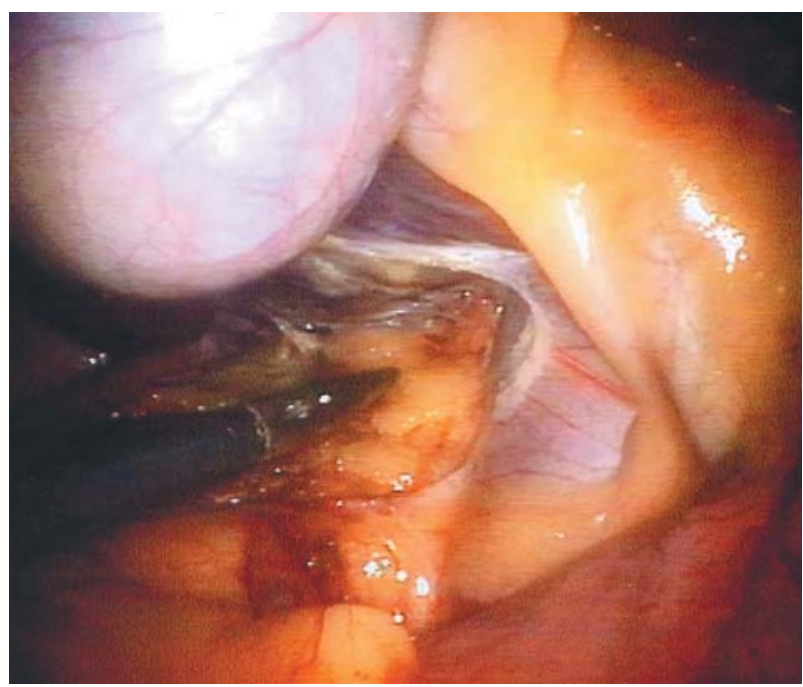

FIGURA 2. Imagen de inicio de la disección en el lado derecho.

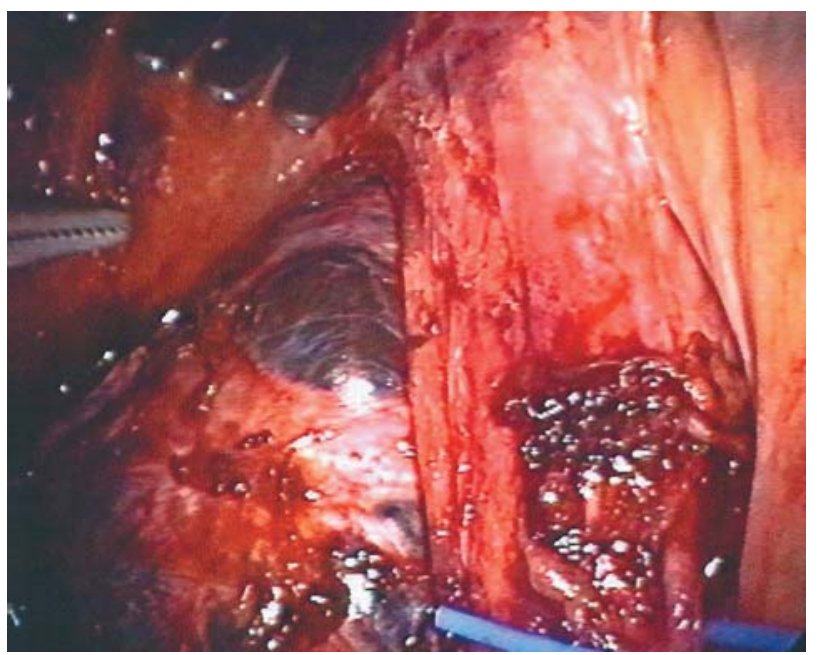

FIGURA 3. Imagen de tumoración en suprarrenal derecha, señalada por las pinzas. 


\section{PACIENTES}

Desde Enero de 1994 hasta Diciembre de 2003, un total de 64 pacientes han sido seleccionados para cirugía laparoscópica por presentar patología suprarrenal, 58 de ellos supuestamente benigna y 6 con sospecha diagnóstica de malignidad. La cirugía se llevó a cabo en el Hospital de León y en la Obra Hospitalaria Nuestra Señora de Regla de León.

Las indicaciones de la adrenalectomía laparoscopica en los pacientes con sospecha diagnóstica de malignidad han sido lesiones de pequeño tamaño, menores de $9 \mathrm{~cm}$, sin signos de invasión ni afectación de las estructuras que le rodean, con márgenes delimitados. En las sospechas de lesiones metastásicas, que estas fueran únicas y que pudiera hacerse cirugía exerética del tumor principal. Se consideró contraindicación de laparoscopia la existencia de cirugías previas en la zona que rodeaba la suprarrenal.

Los 6 pacientes con suprarrenales intervenidas con sospecha de carcinoma tenían una edad media de 68 años (rango 52-78 años), siendo la mitad hombres y la otra mitad mujeres. 2 se localizaron en el lado derecho y 4 en la suprarrenal izquierda. Todas las lesiones se estudiaron por TAC multicorte y ecografía abdominal y en 3 casos fue necesaria la resonancia magnética.

\section{RESULTADOS}

De los 6 pacientes con sospecha diagnóstica de malignidad por TAC y/o RM, en 2 casos resultaron no ser tales sino que se diagnosticaron como adenoma de Conn y el otro caso como hiperplasia nodular tipo Cushing. El diagnóstico de los 4 casos restantes fue en 2 casos de metástasis de adenocarcinoma de pulmón, en un caso hipernefroma metástasis de contralateral y en otro un carcinoma primario de suprarrenal.

El tiempo medio operatorio fue de 120 minutos (rango 85-160 min). El tamaño medio de la glándula tumoral fue de $6 \mathrm{~cm}$ (rango $4-9 \mathrm{~cm}$ ). La estancia media hospitalaria postoperatoria fue de 3 días (rango 2-5 días). No hubo ninguna conversión de laparoscopia a laparotomía y tampoco hubo mortalidad. Tampoco se observaron complicaciones ni intra ni postoperatorias. No se precisó transfusión en ninguno de los pacientes. No hubo necesidad de reintervenciones. Durante el seguimiento de los pacientes, con una media de 24 meses (rango 12-48 meses) no se observó recidiva en ninguno de ellos.

\section{DISCUSIÓN}

Aunque la adrenalectomía abierta es el estándar para la patología adrenal, el gran dolor postoperatorio y la larga convalecencia ha favorecido el uso de la laparoscopia. La adrenalectomía laparoscópica es un proceso eficaz, seguro y mínimamente invasivo ${ }^{4,5,6}$. La mayor experiencia ha aumentado las indicaciones de adrenalectomía laparoscópica. Aunque existe la aceptación generalizada de laparoscopia en tumores benignos, no existe acuerdo respecto al acceso mínimamente invasivo de carcinomas adrenales. Godellas y Prinz recomiendan encarecidamente no operar por via laparoscópica los tumores que se sospeche sean malignos ${ }^{7}$. Indican que la laparoscopia puede ser interesante par descartar metástasis pero las oportunidades de cura son mayores en la disección inicial en bloque y esto se consigue mejor por cirugía abierta. Sin embargo otros autores indican que en lesiones confinadas a la glándula y en la que se observan los principios de cirugía oncológica la evolución de los pacientes es similar a la cirugía abierta, pero con una menor morbilidad ${ }^{3,6,8}$.

El principal criterio para diferenciar si una lesión es benigna o maligna es su tamaño, las lesiones mayores de $6 \mathrm{~cm}$ tienen una mayor posibilidad de ser cancerígenas ${ }^{9}$, así como la no homogeneidad de la lesión en el TAC y una elevación de los niveles de DHEA o estradiol ${ }^{10,11}$.

Existen muy pocos casos en la literatura sobre la adrenalectomía laparoscópica en lesiones cancerosas primarias o metastásicas ${ }^{12,13,14}$. Esto unido a la dificultad de diagnóstico de la lesión adrenal como cancerosa por TAC o RMN cuando está confinada a la glándula y el haberse descrito casos de diseminación del cáncer tras laparoscopia, hace que existan dudas sobre la seguridad de la adrenalectomía laparoscópica en el tratamiento de las lesiones cancerosas adrenales primarias ${ }^{12,15}$. En el caso de metástasis de un cancer confinado a la adrenal, cuando la operación es potencialmente curativa, la posibilidad de adrenalectomía laparoscópica ha de considerarse $^{13,14}$. 
Nuestra experiencia es de seis pacientes en los que se sospechaba una lesión maligna previa a la laparoscopia, confirmándose posteriormente en 4 de ellos. La cirugía en estos pacientes al estar la lesión confinada a la glándula adrenal no se diferenció de las de patología benigna, con un tiempo quirúrgico similar, de 120 min de media frente a los 110 de toda la serie de 64 adrenalectomías laparoscópicas. Las pérdidas sanguíneas fueron mínimas, de 80 a 250 cc de sangre, no precisando ninguno de los pacientes transfusión. Tampoco observamos complicaciones intra ni postoperatorias y los pacientes se recuperaron de la intervención rápidamente, estuvieron ingresados postintervención 3 días de media (de 2 a 4 días de rango). La evolución de estos pacientes fue de no recidiva durante el tiempo de seguimiento, con una media de seguimiento de 24 meses (rango 12 a 48 meses). Todo lo anterior indica, al menos en nuestra serie, que ha habido una evolución similar de la patología de base que de los intervenidos por vía abierta, pero con la gran ventaja de ser una cirugía menos agresiva y con una gran tolerancia para el paciente, por lo que pensamos que en casos seleccionados, en los que la lesión está confinada a la glándula y la finalidad de la intervención es curativo, tanto de neoplasias primarias como de metastásicas, la adrenalectomía laparoscópica ha de considerarse dentro de las opciones terapéuticas por las grandes ventajas que presenta para el paciente sobre la cirugía convencional.

\section{CONCLUSIONES}

La adrenalectomía laparoscópica es una técnica segura y de una gran aplicabilidad en toda la patología suprarrenal benigna. Aunque se precisa de un mayor número de pacientes para una conclusión definitiva, en nuestra serie, cuando la sospecha preoperatoria es de malignidad, sin infiltración de órganos adyacentes el beneficio de la laparoscopia en cuanto a confort postoperatorio se mantiene y no empeora el pronóstico de estos pacientes.

\section{REFERENCIAS}

1. Gagner M, Lacroix A, Bolté E. laparoscopic adrenalectomy in Cushing's sindrome and pheocromocytoma. N. Engl. J. Med 1992;327:1033-1035.

2. Janetscheck G, Altarac S, Finkenstedt G, Passer R, Bartsch G. Technique and results of laparoscopic adrenalectomy. Eur Urol 1996;30:475-479.

3. Kumar U, Albala DM. Laparoscopic approach to adrenal carcionma. J Endourol 2001;15:339-343.

4. Suzuki K, Kageyama S, Ueda D, Ushiyama T, Kawave K, Tajima A, Aso Y. Laparoscopic adrenalectomy: clinical experience with 12 cases. J Urol 1993;150:1099-1102.

5. Porpiglia F, Garrone C, Giraudo G, Destefanis P, Fontana D, Morino M. transperitoneal laparoscopic adrenalectomy: Experience in 72 procedures. J. Endourol. 2001;15: 275279.

6. Assalia A, Gagner M. Laparoscopic adrenalectomy. Br J Surg 2004;91:1259-1274.

7. Godellas CV, Prinz RA. Surgical approaches to adrena neoplasm: Laparoscopic versus open adrenalectomy. Surg Oncol Clin North Am 1998;7:807-817.

8. Sturgeon C, Kebebew E. laparoscopic adrenalectomy for malignancy. Surg Clin North Am 2004;84:755-774.

9. Belldegrun A. Hussain S, Seltzer SE, Loughin KR, Gittes $\mathrm{RF}$, Richie JP. Incidental discovered mass of adrenal gland. Surg Gynecol Obstet 1986;163:203-208.

10. Terzolo M, Ali A, Osella G, Mazza E. Gruppo Piemontese Incidentalomi Surrenalici: Prevalence of adrenal carcinoma among incidentally discovered adrenal masses. Arch Surg 1997; 132:914-919.

11. Ross NS, Aron DC. Hormonal evaluation of the patient with an incidentally discovered adrenal mass. N Eng J Med 1990;323:1401-1405.

12. Heniford BT. Arca MJ, Walsh RM, Gill IS. Laparoscopic adrenalectomy for cancer. Semin Surg Oncol 1999;16:293306.

13. Elashry OM, Clayman RV, Soble JJ, McDougall EM. Laparoscopic adrenalectomy for solitary metachronous contralateral adrenal metastasis form cell carcinoma. J Urol 1997; 157:1217-1222.

14. Tsuji Y, Yasuhuku M, Haryu T, Watanabe Y, Ataka K, Okada M. Laparoscopic adrenalectomy for solitary metachronous adrenal metastasis from lung cancer. Report of a case. Surg Today 1999;29:1277-1279.

15. Hamori E, Meurisse M, Defechereus T. Is laparoscopic resection of a malignant corticoadrenaloma feasible?. Case report of early diffuse and massive peritoneal recurrence alter attempted laparoscopic resection. Ann Chir 1998;52: 364-368.

Dr. A. Vega Vega

Gran Vía de San Marcos, 42 - 6-B

24002 León

(Trabajo recibido el 13 diciembre de 2004) 